\section{Waveguide bandstop filter based on irises and double corrugations for use in industrial microwave ovens}

\author{
F. J. Clemente-Fernández, J. Monzó-Cabrera, J. M. Catalá- \\ Civera, J. L. Pedreño-Molina, A. J. Lozano-Guerrero and A. \\ Díaz-Morcillo
}

\begin{abstract}
In this work it is shown that one of the most employed filtering systems for use in continuous-flow industrial microwave ovens, namely doubly corrugated filters, does not work properly for many values of the electric permittivity of the processed material when it is partially filled. Thus, a novel bandstop filter which solves these inconveniences, based on a combination of metallic irises and double corrugations, is designed by means of CST Microwave Studio ${ }^{\circledR}$ and experimentally validated.
\end{abstract}

Introduction: Corrugated reactive filters [1] are one of the most employed choices that have been proposed in technical when dealing with the leakage problem in continuous-flow industrial microwave ovens that employ open-ended waveguide ports. Singly and doubly corrugated structures have been studied in $[2,3]$ with no material inside the filter. Results when designing bandstop filters in this case may be different from those obtained when a slab of some material is inside the structure. In this work, the influence that the electric permittivity of the processed sample has on the response of doubly corrugated filters when they are partially filled is studied, showing the limitations of these structures depending on the material. In order to solve these inconveniences, a new patented waveguide bandstop filter based on a combination of irises and posts is proposed [4].

Description of the filters under study and experimental setup: The structures under consideration are the conventional doubly corrugated filter and the new proposed filter that are shown in Figs. $1 a$ and $b$, respectively. Both filters have been manufactured by using a WR-340 waveguide with an $8.64 \mathrm{~cm} \times 4.32 \mathrm{~cm}$ cross section. For the doubly corrugated filter the main design parameters are $m$ and $n$, which represent the number of total posts, $b$ and $l$, which determine the size of the corrugations, $d$, which controls the height of the posts, $g$, which determines the height of the aperture where the material goes through, and $w$, which represents the width of the open port. Regarding the new filter the main design parameters are $b_{x}$ and $b_{z}$, which control the separation between posts in the two directions $x$ and $z, D$, the diameter of the posts, $d_{p o s t}$, the height of each post, $d_{i r i s}$, the height of the metallic irises, and $m, n, g$ and $w$ as explained earlier. The thickness of the irises is $2 \mathrm{~mm}$ and they are placed exactly in the middle between the rows of posts. In this case, when optimizing this filter the parameters which are modified are $g, D, d_{\text {post }}$ and $d_{i r i s}$.

Two monomode prototypes have been manufactured. Their geometry is shown in Table 1. These filters are connected to a Rohde \& Schwarz ZVA67 VNA by using a WR-340 coaxial-to-waveguide transition. In this way, the $S_{21}$ parameter is obtained from 2 to $3 \mathrm{GHz}$. The behaviour of the filters is also tested when three different pieces of marble $\left(\varepsilon_{r 1}=\right.$ $\left.7.90-\mathrm{j} 0.3476 ; \varepsilon_{r 2}=8.18-\mathrm{j} 0.1603\right)$ and granite $\left(\varepsilon_{r 3}=5.49-\mathrm{j} 0.3190\right)$ are placed inside of them.

Design and optimization procedures: CST Microwave Studio ${ }^{\circledR}[5]$ has been used to perform the simulations by using the time domain solver from 2 to $3 \mathrm{GHz}$. The main goal was centering the stopband at 2.45
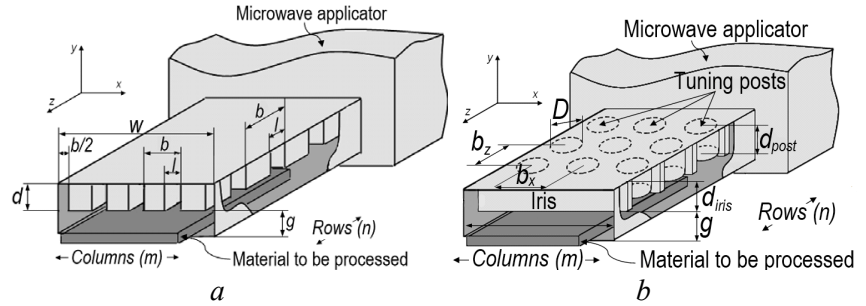

Fig. 1 Waveguide bandstop filters under study.

$a$ Doubly corrugated filter

$b$ New proposed filter
Table 1: Parameters for the manufactured filters

\begin{tabular}{|c|c|c|c|}
\hline \multicolumn{2}{|c|}{ Doubly corrugated filter } & \multicolumn{2}{c|}{ New proposed filter } \\
\hline Parameter & Value & Parameter & Value \\
\hline Posts $(m \times n)$ & $3 \times 4$ & Posts $(m \times n)$ & $3 \times 4$ \\
\hline$g$ & $2 \mathrm{~cm}$ & $g$ & $3.307 \mathrm{~cm}$ \\
\hline$d$ & $2.839 \mathrm{~cm}$ & $d_{\text {iris }}$ & $2.3 \mathrm{~cm}$ \\
\hline$l$ & $2.879 \mathrm{~cm}$ & $d_{\text {post }}($ Tunable $)$ & $2.61 \mathrm{~cm}$ \\
\hline$b$ & $1.6 \mathrm{~cm}$ & $D$ & $1.2 \mathrm{~cm}$ \\
\hline & & $b_{x}$ & $2.867 \mathrm{~cm}$ \\
\hline & & $b_{z}$ & $3.225 \mathrm{~cm}$ \\
\hline
\end{tabular}

$\mathrm{GHz}$, where typical industrial magnetrons work. Attenuations around 60 $\mathrm{dB}$ are usually enough to guarantee safe levels of leaked energy.

In order to optimize the new bandstop filter, an optimization procedure in MATLAB $B$ by means of genetic algorithms has been employed. 50 generations and 50 individuals per generation have been used. An evaluation function $F$ is defined in (1) as the sum of differences between the value of the $S_{21}$ parameter (if greater than -60 $\mathrm{dB}$ ) and the value of $-60 \mathrm{~dB}$, from 2.44 to $2.46 \mathrm{GHz}$.

$$
F=\sum\left[\left|S_{21}\left(f_{i}\right)\right|-(-60 \mathrm{~dB})\right], \forall i|| S_{21}\left(f_{i}\right) \mid>-60 \mathrm{~dB}
$$

where $\left|S_{21}\right|$ is the magnitude of the transmission coefficient and $i$ is the index for each frequency point within the optimized frequency range.

Behaviour of traditional filters: In order to study the influence of the complex permittivity of the material within the filter, a 2-D parametric sweep simulation has been performed by varying both the dielectric constant and the loss tangent of the sample. The geometry of the simulated structure is the one indicated in Table 1 for the doubly corrugated filter. The sample is placed covering from the first to the last row of posts. Results of the transmission coefficient for the $\mathrm{TE}_{10}$ mode at $2.45 \mathrm{GHz}$ are shown in Fig. 2. It can be observed that $\varepsilon$ ' values under 4 show valid results, regardless the value of $\tan \delta$. However, for values of $\varepsilon^{\prime}$ associated with the samples greater than 4 the attenuation decreases to unacceptable levels. Only when both the loss tangent and the dielectric constant reach high values the attenuation becomes acceptable again. Therefore, there is a wide range of $\left(\varepsilon^{\prime}, \tan \delta\right)$ pairs where the filter cannot be used with safety. The three materials under study have been experimentally tested by placing them inside the doubly corrugated filter. The transmission coefficient for these materials is shown in Fig. 3, confirming the inability to deal with them. Genetic algorithms have been employed trying to improve the performance of the filter with these materials by optimizing all the design parameters. The main conclusion is that it is not possible to obtain a doubly corrugated filter which works properly with this kind of materials. This is due to the increase of the effective height of the aperture where the material goes through, which makes impossible to filter the $\mathrm{TE}_{10}$ mode with only the metallic posts.

Behaviour of new proposed filters: A novel filter based on a combination of metallic irises and tuning posts has been designed. An optimization procedure based on genetic algorithms has been applied by varying the parameters $D, d_{i r i s}, d_{\text {post }}$ and $g$ when the filter is empty according to the evaluation function in (1). The final parameters are shown in Table 1. Fig. 4 shows a comparison between simulation and measurements when no material is placed inside the filter, showing a good agreement. When introducing the pieces of marble and granite, a readjustment of the length of the tuning posts must be done, since the dielectric properties of each material are different. Therefore, the structure was optimized again by taking into account each material and, after a manual tuning process, it was possible to obtain excellent attenuation results at the frequency of interest for the three materials as shown in Fig. 5. From Figs. 4 and 5 one can conclude that this new architecture works properly when it is empty and partially filled with a material. In fact, the attenuation of this new filter is $50 \mathrm{~dB}$ higher than the one measured in doubly corrugated filters for the same materials.

Conclusion: It has been shown that doubly corrugated filters do not work properly when dealing with a wide range of materials. Thus, a new filter based on a combination of metallic irises and tuning posts has 
been designed, simulated and experimentally validated. Results show that it is possible to obtain good attenuation values with this structure for both the empty filter and when filled with materials. The measured attenuation levels with this new filter when partially filled are $50 \mathrm{~dB}$ higher than the ones measured for the traditional doubly corrugated filter for the used materials. Moreover, using tuning posts makes the structure much more flexible, allowing us to deal with different materials with the same base filter.

Acknowledgments: This work was supported in part by the Fundación Séneca under the fellowship 16381/FPI/10 and the project 11689/PI/09.

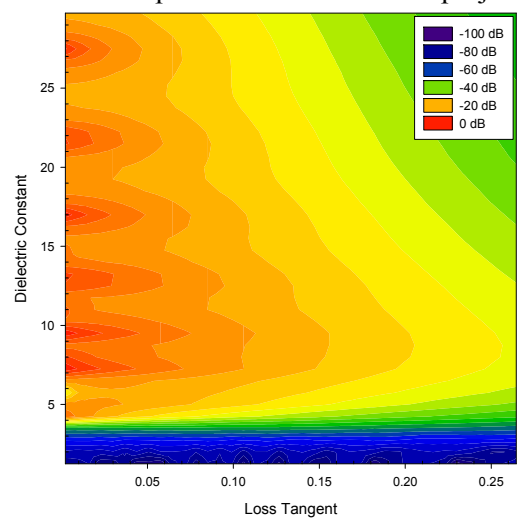

Fig. 2 Transmission coefficient vs. dielectric constant and loss tangent of the processed material for the doubly corrugated filter.

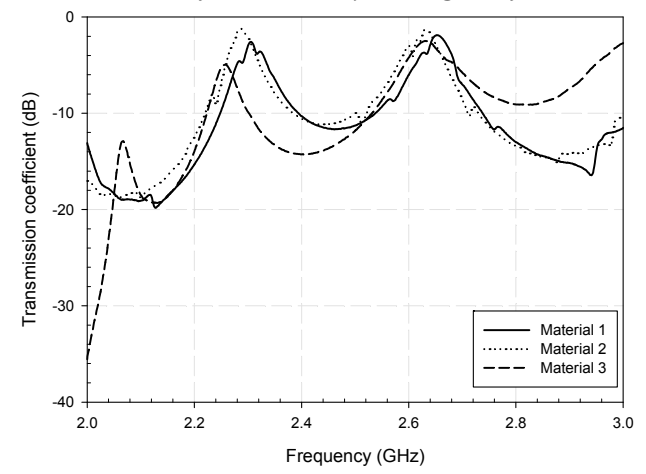

Fig. 3 Measured transmission coefficient for the doubly corrugated filter with the materials under test.

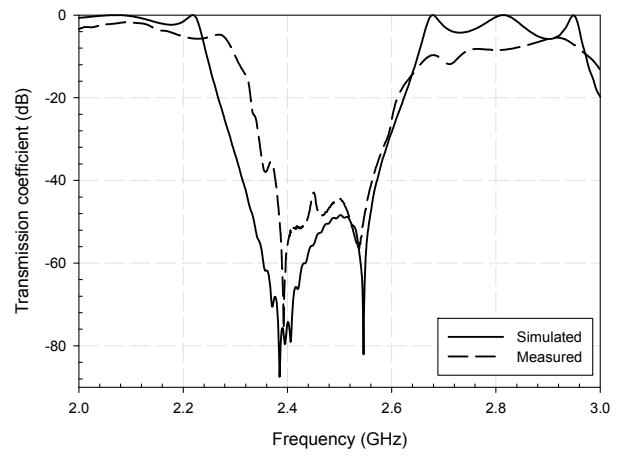

Fig. 4 Comparison between simulation and measurements for the new filter with no material inside $\left(d_{\text {post }}=2.61 \mathrm{~cm}\right)$.

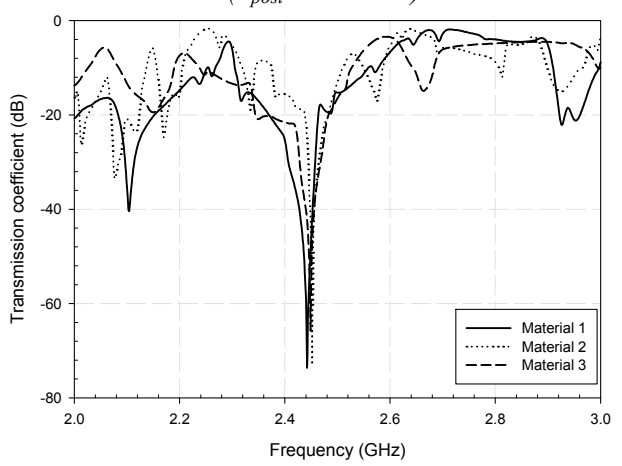

Fig. 5 Measured transmission coefficient for the new proposed filter with the materials under test.
F. J. Clemente-Fernández, J. Monzó-Cabrera, J. L. Pedreño-Molina, A. J. Lozano-Guerrero and A. Díaz-Morcillo (Dpto. de Tecnologías de la Información y Comunicaciones, Univ. Politécnica de Cartagena, E30202, Cartagena, Murcia, Spain)

E-mail: francisco.clemente@upct.es

J. M. Catalá-Civera (Dpto. de Comunicaciones, Univ. Politécnica de Valencia, E-46022, Valencia, Spain)

\section{References}

1 Vankoughnett, A.L., and Dunn, J.G.: 'Doubly corrugated chokes for microwave heating systems', J. Microw. Power, 1973, 8, (1), pp. 101110

2 Soto, P., Boria, V.E., Catalá-Civera, J.M., Chouaib, N., Guglielmi, M., and Gimeno, B.: 'Analysis, design, and experimental verification of microwave filters for safety issues in open-ended waveguide systems', IEEE Trans Microwave Theory Tech, 2000, 48, (11), pp. 2133-2140

3 Catalá-Civera, J.M., Soto, P., Boria, V.E., Balbastre, J.V., and de los Reyes, E.: 'Design Parameters of Multiple Reactive Chokes for Open Ports in Microwave Heating Systems, Advances in Microwave and Radio Frequency Processing' (Springer, Berlin, 2006)

4 Monzó-Cabrera, J., Pedreño-Molina, J. L., Díaz-Morcillo, A., Clemente-Fernández, F. J., Díaz-García, J., Baños-Tudela, J. M., and Méndez-Hernández, P. A.: 'Filtro multimodo de microondas abierto en tecnología de guía de onda mediante la combinación de postes e iris para hornos de calentamiento por microondas', ES2337756 Patent, 2011

5 CST STUDIO SUITETM, http://www.cst.com 